\title{
Moderate influenza vaccine effectiveness in Victoria, Australia, 2011
}

J E Fielding (james.fielding@mh.org.au) ${ }^{1,2}$, K A Grant ${ }^{1}$, T Tran $^{1}$, H A Kelly ${ }^{1,2}$

1. Victorian Infectious Diseases Reference Laboratory, Melbourne, Australia

2. National Centre for Epidemiology and Population Health, The Australian National University, Canberra, Australia

We used a sentinel general practitioner (GP) network to conduct surveillance for laboratory-confirmed influenza amongst patients presenting with influenza-like illness (ILI) in Victoria, Australia in 2011. The testnegative variation of the case control study design was used to estimate effectiveness for seasonal trivalent influenza vaccine. Cases and controls were ILI patients that tested positive and negative for influenza, respectively. Vaccination status was recorded by GPs and vaccine effectiveness (VE) was calculated as (1-adjusted odds ratio)x100\%. There were 529 patients included in the study, of which $29 \%$ were influenzapositive. Twelve percent of study participants were reported as vaccinated, $6 \%$ of cases and $15 \%$ of controls. Adjusted VE against all influenza was $56 \%$, but not statistically significant. There was generally little variation in VE estimates when stratified by virus type and subtype, which is consistent with good matches between circulating strains and the vaccine strains. The VE was higher among adults of working age than among children.

\section{Introduction}

Victoria accounts for approximately $25 \%$ of Australia's population of 23 million people. It has a temperate climate, and the influenza season usually occurs between June and October. Each season, the Victorian Infectious Diseases Reference Laboratory uses a network of sentinel general practitioners (GPS) to conduct surveillance for influenza-like illness (ILI) and laboratory-confirmed influenza. The system has been operational since 1998, with an average of 60 GPs participating each year. This surveillance system is used to estimate vaccine effectiveness (VE) of the seasonal influenza vaccine.

Seasonal influenza vaccination in Australia is a publicly funded programme. The Australian government provides free influenza vaccination to all Australians aged 65 years and older, Aboriginal and Torres Strait Islander people over 15 years of age, pregnant women and individuals aged six months and older with medical conditions predisposing to severe influenza [1].
Individuals may also be vaccinated outside the funded programme, such as through workplaces. The influenza virus composition of the seasonal trivalent influenza vaccine (TIV) in Australia in 2011 was A/ California/7/2009 (H1N1)-like virus, A/Perth/16/2009 $\left(\mathrm{H}_{3} \mathrm{~N}_{2}\right)$-like virus, and $B /$ Brisbane/6o/2008-like virus (of the B/Victoria/2/87 lineage) [2].

Here we use the results from laboratory-confirmed influenza surveillance in Victoria to estimate TIV effectiveness in 2011 using the prospective test-negative variation of the case control study. This design has been used in Europe, North America and Australia [3-6]. We aimed to calculate type- and subtype-specific VE estimates and used them in combination with surveillance data to make inferences how well the 2011 seasonal TIV matched circulating strains. The strain composition recommended for use in the 2011 southern hemisphere influenza vaccine was the same as the one subsequently used in the $2011 / 12$ northern hemisphere seasonal vaccine [7].

\section{Methods}

In 2011, 97 GPs participated in the surveillance system which operated from 2 May to 30 October inclusive. Advertising in GP circulars was used to encourage GPs to participate in the programme and targeted recruitment was undertaken in geographical areas considered to be poorly represented. A relatively even and widespread distribution suggested adequate representation of the 97 GPs throughout the metropolitan and most rural areas of the state. GPs reported the total number of consultations per week from which proportions were calculated as the number of ILI patients per 1,000 consultations. ILI was defined as fever (or history of fever), cough, and either fatigue or malaise [8]. GPs were asked to collect a nose and/or throat swab from patients with an ILI within four days of the onset of the patient's symptoms and provide data on the patient's age, sex, date of symptoms onset, influenza vaccination status in 2011 and 2010, date of vaccination and presence of comorbid conditions for which influenza 
vaccination is indicated. Patients were chosen for swabbing at the discretion of the GP.

To test for influenza viruses, RNA was extracted from clinical specimens using a Corbett extraction robot followed by reverse transcription using random hexamers. cDNA was amplified using an ABI-7500 Fast Real-Time PCR System incorporating primers and probes specific for the detection of type A, B and C influenza viruses. Samples that tested positive for influenza type $A$ in this assay were subtyped in a second real-time PCR assay incorporating primers and probe specific for influenza $A\left(\mathrm{H}_{1} \mathrm{~N}_{1}\right)$ pdmo9, $A\left(\mathrm{H}_{1}\right)$ (non-pandemic) and $A\left(\mathrm{H}_{3}\right)$ haemagglutinin genes.

VE was defined as (1-adjusted odds ratio)x100\%, where the odds ratio is the ratio of odds of laboratory-confirmed influenza cases being vaccinated to the odds of controls (those that tested negative for influenza) being vaccinated. Logistic regression was used to calculate odds ratios and $95 \%$ confidence intervals that were adjusted for the variables of age group, month of specimen collection and comorbidity. There was not sufficient statistical power to generate age-specific VE estimates for the age group $\geq 65$ years or to further stratify the age group of 0-19 year-olds. Patients were excluded from the VE analysis if vaccination status was unknown, if the date of symptom onset was unknown or if there was an interval greater than four days between symptom onset and specimen collection, based on the decreased likelihood of a positive result after this time $[9,10]$. Patients were considered not vaccinated if there was less than 14 days between the date of vaccination and symptom onset. All analyses were conducted using Stata (version 10.0; StataCorp LP). The chi-squared test was used to compare proportions, with p<0.05 considered statistically significant.

\section{Results}

Participating GPs reported seeing a total of 194,295 patients during the reporting period, of whom 945 (0.5\%) met the ILI case definition, a proportion that was consistent with previous years. As the reporting of ILI cases is not identifiable and separate to those who are swabbed (for whom data are recorded on a laboratory test request form), we are unable to assess any demographic or vaccination status differences between those who were swabbed and those who were not. Of the $945 \mathrm{ILI}$ cases, 665 (70\%) were swabbed and 185 (28\%) tested positive for influenza. In general, influenza $A\left(\mathrm{H}_{1} \mathrm{~N}_{1}\right)$ pdmog predominated during the first half of the season, $\mathrm{A}\left(\mathrm{H}_{3}\right)$ during the middle to latter part, whilst cases of influenza B were detected throughout (Figure). One case of influenza type $C$ infection was also detected.

We excluded 136 swabbed patients (20\%) from the VE analysis due to unknown vaccination status $(n=25)$, unknown date of symptom onset $(n=44)$ or more than four days between symptom onset and specimen

\section{FIGURE}

Influenza-positive and -negative patients at sentinel general practices by week, Victoria, 2 May to 30 October ( $\mathrm{n}=665$ )

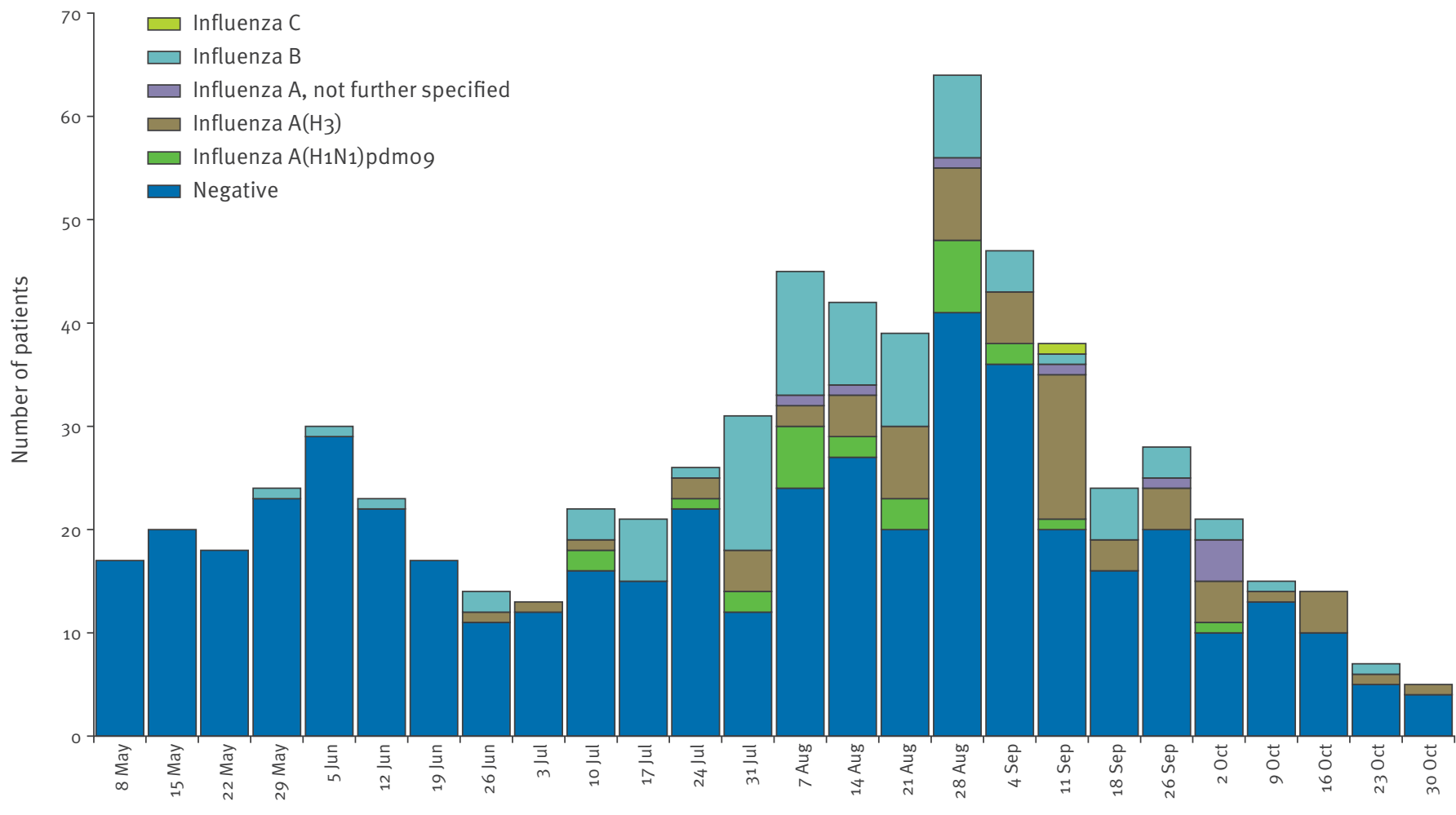

Week 2011 (weeks ending on given date) 
collection $(n=80)$; some were excluded for more than one reason. The case of influenza type $C$ infection was also excluded. There was no statistically significant difference between the swabbed patients that were included and those that were excluded from the study by vaccination status $(p=0.11)$, influenza positivity $(p=0.07)$, age group $(p=0.72)$, presence of a comorbid condition $(p=0.21)$ or vaccination in $2010(p=0.10)$.

Of the 529 patients included in the study, $155(29 \%)$ were cases and 374 (71\%) were controls. Cases were significantly younger than controls $(p=0.004)$ and more common in August and September ( $p<0.001$ ), but there was no statistically significant difference between cases and controls by sex $(p=0.31$ ) (Table 1). There was no statistically significant difference between cases and controls with respect to presence of a comorbidity recommended for influenza vaccination $(p=0.15)$, although those with a comorbid condition were more likely to be older ( $p<0.001)$ and to be vaccinated ( $p<0.001)$. Being vaccinated in 2010 was not associated with testing positive for influenza $(p=0.21)$, but was associated with older age ( $p<0.001)$ and with vaccination in 2011 ( $p<0.001)$.

Of the 529 patients eligible for the VE analysis, 65 $(12 \%)$ were reported as vaccinated, with a statistically significant difference between cases $(6 \%)$ and controls $(15 \%)(p=0.008)$ (Table 2). No cases of influenza $A\left(H_{1} N_{1}\right) p d m o 9$ were reported as vaccinated. The proportion vaccinated was significantly higher in older age groups (p<0.001), but there was no statistically

\section{TABLE 1}

Characteristics of cases and controls, vaccine effectiveness study, Victoria, 2 May to 30 October $(\mathrm{n}=529)$

\begin{tabular}{|c|c|c|c|}
\hline & $\begin{array}{l}\text { Number of } \\
\text { controls (\%) }\end{array}$ & $\begin{array}{l}\text { Number of } \\
\text { cases (\%) }\end{array}$ & $\mathrm{p}$ value \\
\hline \multicolumn{4}{|l|}{ Sex } \\
\hline Female $^{\mathrm{a}}$ & $189(51)$ & $86(55)$ & 0.31 \\
\hline \multicolumn{4}{|l|}{ Age } \\
\hline $0-19$ years & $108(29)$ & $67(43)$ & \multirow{3}{*}{0.004} \\
\hline $20-64$ years & $249(67)$ & $85(55)$ & \\
\hline$\geq 65$ years & $17(5)$ & $3(2)$ & \\
\hline \multicolumn{4}{|l|}{ Month of swab collection } \\
\hline May & $71(19)$ & $2(1)$ & \multirow{6}{*}{$<0.001$} \\
\hline June & $64(17)$ & $1(\ll 1)$ & \\
\hline July & $59(16)$ & $30(19)$ & \\
\hline August & $107(29)$ & $74(48)$ & \\
\hline September & 49 (13) & $39(25)$ & \\
\hline October & $24(6)$ & $9(6)$ & \\
\hline Comorbid condition $^{\mathrm{b}}$ & $43(13)$ & $12(9)$ & 0.15 \\
\hline Previously vaccinated ${ }^{c}$ & $76(22)$ & $25(17)$ & 0.21 \\
\hline Total & 374 & 155 & \\
\hline
\end{tabular}

a No data for one control.

b No data for 50 controls and 15 cases.

No data for 27 controls and seven cases. significant difference between those vaccinated and not vaccinated by month of testing $(p=0.63)$.

There was little difference in the overall crude $(60 \%)$ and adjusted (56\%) point estimates for VE against all influenza, although only the crude estimate was statistically significant (Table 3). Although slightly higher against influenza $A\left(\mathrm{H}_{1} \mathrm{~N}_{1}\right)$ pdmog, age-adjusted VE estimates were generally consistent when stratified by type and subtype, however, $95 \%$ confidence intervals for estimates in the age group of $0-19$ year-olds were very wide. Crude VE against influenza $A\left(\mathrm{H}_{1} \mathrm{~N}_{1}\right)$ pdmog was $100 \%$ because none of 24 cases with confirmed influenza $A\left(\mathrm{H}_{1} \mathrm{~N}_{1}\right)$ pdmog were vaccinated, but the VE was reduced after adjustment.

A sensitivity analysis conducted by restricting inclusion of cases and controls to the influenza season in 2011 when cases are more likely to be detected (the period from 20 June to 30 October when at least one influenza case was detected in consecutive weeks) resulted in changes to the point estimates from $0 \%$ to $1 \%$. Not censoring patients for whom there were more than four days between symptom onset and specimen collection reduced the crude and overall adjusted VE estimates from $0 \%$ to $25 \%$ and from $2 \%$ to $14 \%$, respectively.

\section{Discussion}

Using a population of patients with ILI who consulted sentinel GPs in Victoria, Australia, we have estimated a moderate effectiveness of $56 \%$ for the 2011 seasonal TIV against all influenza, although this was not statistically significant. VE estimates for the age group of 0-19 year-olds (childhood) were lower and considerably less precise than those for the age group of 20-64 yearolds. This is consistent with our observations in previous years which have highlighted the utility of this GP surveillance programme for estimating VE among working age adults who comprise most of the surveillance population $[11,12]$.

\section{TABLE 2}

Number and vaccination status of cases and controls by age group, vaccine effectiveness study, Victoria, 2 May to 30 October $(n=529)$

\begin{tabular}{|l|c|c|c|c|c|}
\hline \multicolumn{2}{|c|}{} & \multicolumn{5}{|c|}{ Age group (years) } \\
\cline { 2 - 6 } \multicolumn{2}{|c|}{ Controls } & $0-19$ & $20-64$ & $\geq 65$ & Total \\
\cline { 2 - 6 } & Vaccinated (\%) & $2(2)$ & $43(17)$ & $10(59)$ & $\mathbf{5 5}(15)$ \\
\hline \multirow{2}{*}{$\begin{array}{l}\text { All influenza } \\
\text { cases }\end{array}$} & $\mathrm{n}$ & 67 & 85 & 3 & $\mathbf{1 5 5}$ \\
\cline { 2 - 6 } & Vaccinated (\%) & $1(1)$ & $6(7)$ & $3(100)$ & $\mathbf{1 0}(6)$ \\
\hline $\begin{array}{l}\text { Influenza } \\
\text { A(H1N1)pdm09 } \\
\text { cases }\end{array}$ & $\mathrm{n}$ & 4 & 20 & 0 & $\mathbf{2 4}$ \\
\cline { 2 - 6 } & Vaccinated (\%) & $0(0)$ & $0(0)$ & $0(0)$ & $0(0)$ \\
\hline $\begin{array}{l}\text { Influenza A(H3) } \\
\text { cases }\end{array}$ & $\mathrm{n}$ & 24 & 29 & 1 & 54 \\
\cline { 2 - 6 } & Vaccinated (\%) & $0(0)$ & $3(10)$ & $1(100)$ & $\mathbf{4}(7)$ \\
\hline $\begin{array}{l}\text { Influenza B } \\
\text { cases }\end{array}$ & $\mathrm{n}$ & 37 & 30 & 2 & 69 \\
\cline { 2 - 6 } & Vaccinated (\%) & $1(3)$ & $1(3)$ & $2(100)$ & $\mathbf{4}(6)$ \\
\hline
\end{tabular}


Strain typing surveillance data suggested good matches to the vaccine strains: $89 \%$ of 87 influenza $\mathrm{A}\left(\mathrm{H}_{1} \mathrm{~N}_{1}\right)$ isolates were $\mathrm{A} / \mathrm{California} / 7 / 2009$-like with the remainder $\mathrm{A} / \mathrm{California/7/2009}$-like (low reactor); $96 \%$ of 122 type $A\left(H_{3} N_{2}\right)$ isolates were $A / P e r t h / 16 / 2009$-like with the remainder $A / P e r t h / 16 / 2009$-like (low reactor); $96 \%$ of 136 type $B$ isolates were $B /$ Brisbane/6o/2008like, $4 \%$ were $B / B$ risbane/60/2008-like (low reactor) and fewer than $1 \%$ were B/Florida/4/2006-like (low reactor) of the $B / Y a m a g a t a / 16 / 88$ lineage (personal communication: K O’Bryan, World Health Organization Collaborating Centre for Reference and Research on Influenza, December 2011). Thus, the type- and subtype-stratified VE point estimates are broadly consistent with a good match to the circulating strains. However, none of the adjusted VE estimates was statistically significant suggesting insufficient study power. This is particularly evident in the childhood age group of the 0-19 year-olds.

To our knowledge there are no other published data for 2011 southern hemisphere seasonal influenza vaccine effectiveness. However, a point of comparison to other studies exists given the strain composition has not changed for the 2010/11 northern hemisphere and 2010 and 2011 southern hemisphere seasonal TIVs. In general the estimates obtained from our study were higher than those from other comparable studies. Using the same method we were able to demonstrate an effectiveness of $89 \%$ for the 2010 TIV against influenza $\mathrm{A}\left(\mathrm{H}_{1} \mathrm{~N}_{1}\right)$ pdmog among working age adults [12], compared with the $78 \%$ effectiveness observed this year. A study conducted amongst inpatients in 15 Australian hospitals in the same period in 2010 estimated a statistically significant effectiveness of $49 \%$ for TIV against hospitalisation with influenza $A\left(\mathrm{H}_{1} \mathrm{~N}_{1}\right)$ pdmo9 [13]. Similarly in Europe, preliminary estimates for seasonal influenza vaccine effectiveness against all influenza using the test-negative variation of the case control study design among ILI patients seen in primary care were lower than our study, ranging from $5 \%$ to $50 \%$ [14-17]. The pooled end-of-season analysis of the European data resulted in lower adjusted estimates of VE against both influenza $A\left(\mathrm{H}_{1} \mathrm{~N}_{1}\right)$ pdmog (27\%) and type B influenza (64\%) in working age adults compared to our study, although neither was statistically significant [18].

In our analysis we attempted to control for variables generally considered to be confounders [19], that is, those assumed to be associated with both exposure (vaccination) and outcome (influenza) but not on the causal pathway. These include age, month of swab collection and presence of a comorbid condition for which influenza vaccine is indicated. We observed generally little variation between crude VE estimates and those adjusted for these confounding variables. Only age was significantly associated with both vaccination and influenza. Month of swab collection and comorbidity were significantly associated with outcome and exposure respectively, but neither was significantly associated with both. Other studies using the same variation of the test-negative case control study as this one have also adjusted for receipt of influenza vaccine within a year before the study $[16,18]$. Whilst we collected this data field in 2011, its inclusion as a covariate in the adjusted model resulted in considerable variation from the crude and the age-, month- and comorbidity-adjusted VE estimates. However, further statistical analysis did not support inclusion of previous vaccination in the model because it assumes that previous vaccination has the same effect regardless of vaccination in the current season, and because of its high degree of correlation with current vaccination status which skews and reduces the precision of the VE estimate.

While variables may be considered to be theoretical confounders they may result in biases that could under- or over-estimate the VE. Results from influenza VE studies in Europe for the 2010/11 season included comments about the need for a cautious approach to dealing with such variables $[17,20]$ and highlight the need for further clarification of the optimal analysis for the test-negative design when used to estimate influenza VE. Whilst relatively new, the method is administratively practical and theoretically acceptable, and

\section{TABLE 3}

Crude and adjusted vaccine effectiveness of seasonal vaccine against influenza by age group and type/subtype, Victoria, 2 May to 30 October $(n=529)$

\begin{tabular}{|l|c|c|c|c|}
\hline \multirow{2}{*}{} & \multicolumn{3}{|c|}{ Influenza vaccine effectiveness (95\% confidence interval) } \\
\cline { 2 - 5 } & \multirow{2}{*}{ Crude } & \multicolumn{2}{c|}{ Adjusted $^{\mathrm{a}}$} \\
\cline { 2 - 5 } & $60(19$ to 80$)$ & $0-19$ years & $20-64$ years & $56(-2$ to 81$)$ \\
\hline Influenza $A\left(\mathrm{H}_{1} \mathrm{~N}_{1}\right)$ pdmo9 & $100(6 \text { to } 100)^{\mathrm{b}}$ & Not defined & $77(-44 \text { to } 100)^{\mathrm{b}, \mathrm{c}}$ & $78(-38 \text { to } 100)^{\mathrm{b}, \mathrm{c}}$ \\
\hline Influenza $\mathrm{A}\left(\mathrm{H}_{3}\right)$ & $54(-34$ to 84$)$ & $-44(-1,757 \text { to } 100)^{\mathrm{b}, \mathrm{c}}$ & $48(-99$ to 86$)$ & $58(-53$ to 89$)$ \\
\hline Influenza B & $64(-2$ to 88$)$ & $-16(-1,298$ to 90$)$ & $78(-77$ to 97$)$ & $53(-68$ to 87$)$ \\
\hline
\end{tabular}

a Adjusted for month of swab collection and comorbidities.

b Calculated using exact method.

c Median unbiased estimates. 
we will continue to refine it in collaboration with other investigators that have adopted it.

As previously discussed, other limitations of the study must also be taken into account when considering the results $[6,12,21]$. Briefly, the study was conducted in a general practice setting and the results are thus representative of the mid-range of the influenza clinical spectrum. Those not sick enough to attend a medical practitioner and more severe cases requiring hospitalisation were not part of the sampling frame. We were unable to quantify immunity from previous infection or healthy vaccinee bias, both of which overestimate VE. Conversely though, when conducted retrospectively, the test-negative case control design generally underestimates true VE under most conditions of test sensitivity, specificity and the ratio of influenza to noninfluenza attack rates [22].

Overall, the seasonal TIV was moderately effective against medically attended influenza in Victoria, Australia during the 2011 southern hemisphere season. These VE estimates were generally consistent among working age adults when stratified by type and influenza A subtype, and consistent with an apparent good match between TIV and circulating strains during a season which saw the re-emergence of the influenza $\mathrm{A}\left(\mathrm{H}_{3} \mathrm{~N}_{2}\right)$ subtype [23].

\section{Acknowledgments}

We thank Sheena Sullivan from the World Health Organization Collaborating Centre for Reference and Research on Influenza, Melbourne, Australia and Nevil Pierse from the Department of Public Health, University of Otago, Wellington, New Zealand for statistical advice. We especially thank the general practitioners that participated in the surveillance system during 2011 and staff of the Viral Identification Laboratory at the Victorian Infectious Diseases Reference Laboratory who conducted the influenza testing. The sentinel general practice surveillance programme is funded by the Victorian Government Department of Health.

\section{References}

1. Australian Government Department of Health and Ageing. Influenza (Flu). Canberra: Department of Health and Ageing [Accessed Dec 15 2011]. Available from: http://health.gov.au/ internet/immunise/publishing.nsf/Content/immunise-influenza

2. Recommended viruses of influenza vaccines for use in the 2011 influenza season (southern hemisphere). Wkly Epidemiol Rec. 2010;85(41):402-12.

3. Kissling E, Valenciano $M$, Falcao J, Larrauri A, Widgren $\mathrm{K}$, Pitigoi D, et al. "I-MOVE" towards monitoring seasonal and pandemic influenza vaccine effectiveness: lessons learnt from a pilot multi-centric case-control study in Europe, 2008-9. Euro Surveill. 2009;14(44):pii=19388. Available from: http://www. eurosurveillance.org/ViewArticle.aspx?Articleld =19388

4. Belongia EA, Kieke BA, Donahue JG, Coleman LA, Irving SA, Meece JK, et al. Influenza vaccine effectiveness in Wisconsin during the 2007-08 season: comparison of interim and final results. Vaccine. 2011;29(38):6558-63.

5. Skowronski DM, De Serres G, Crowcroft NS, Janjua NZ, Boulianne N, Hottes TS, et al. Association between the 2008o9 seasonal influenza vaccine and pandemic $\mathrm{H}_{1} \mathrm{~N}_{1}$ illness during Spring-Summer 2009: four observational studies from Canada. PLoS Med. 2010;7(4):e1000258.

6. Fielding JE, Grant KA, Papadakis G, Kelly HA. Estimation of type- and subtype-specific influenza vaccine effectiveness in
Victoria, Australia using a test negative case control method, 2007-2008. BMC Infect Dis. 2011;11:170.

7. Recommended composition of influenza virus vaccines for use in the 2011-2012 northern hemisphere influenza season. Wkly Epidemiol Rec. 2011;86(10):86-90.

8. Thursky K, Cordova SP, Smith D, Kelly H. Working towards a simple case definition for influenza surveillance. J Clin Virol. 2003;27(2):170-9.

9. Carrat F, Vergu E, Ferguson NM, Lemaitre M, Cauchemez S, Leach S, et al. Time lines of infection and disease in human influenza: a review of volunteer challenge studies. Am J Epidemiol. 2008;167(7):775-85.

10. Cowling BJ, Chan KH, Fang VJ, Lau LL, So HC, Fung RO, et al. Comparative epidemiology of pandemic and seasonal influenza A in households. N Engl J Med. 2010;362(23):2175-84.

11. Kelly H, Carville K, Grant K, Jacoby P, Tran T, Barr I. Estimation of influenza vaccine effectiveness from routine surveillance data. PLoS One. 2009;4(3):e5079.

12. Fielding JE, Grant KA, Garcia K, Kelly HA. Effectiveness of seasonal influenza vaccine against pandemic ( $\left.\mathrm{H}_{1} \mathrm{~N}_{1}\right) 2009$ virus, Australia, 2010. Emerg Infect Dis. 2011;17(7):1181-7.

13. Cheng AC, Kotsimbos T, Kelly HA, Irving LB, Bowler SD, Brown $\mathrm{SG}$, et al. Effectiveness of $\mathrm{H}_{1} \mathrm{~N}_{1} / \mathrm{og}_{\text {monovalent }}$ and trivalent influenza vaccines against hospitalization with laboratoryconfirmed $\mathrm{H}_{1} \mathrm{~N}_{1} / 09$ influenza in Australia: a test-negative case control study. Vaccine. 2011;29(43):7320-5.

14. Kissling E, Valenciano M. Early estimates of seasonal influenza vaccine effectiveness in Europe, 2010/11: I-MOVE, a multicentre case-control study. Euro Surveill. 2011;16(11): $p=19818$. Available from: http://www.eurosurveillance.org/ViewArticle. aspx?Articleld $=19818$

15. Pebody R, Hardelid P, Fleming DM, McMenamin J, Andrews N, Robertson C, et al. Effectiveness of seasonal 2010/11 and pandemic influenza $A\left(\mathrm{H}_{1} \mathrm{~N}_{1}\right) 2009$ vaccines in preventing influenza infection in the United Kingdom: mid-season analysis 2010/11. Euro Surveill. 2011;16(6):pii=19791 Available from: http://www.eurosurveillance.org/ViewArticle. aspx?Articleld=19791

16. Savulescu C, Jimenez-Jorge S, de Mateo S, Ledesma J, Pozo F, Casas I, et al. Effectiveness of the $2010 / 11$ seasonal trivalent influenza vaccine in Spain: preliminary results of a case-control study. Euro Surveill. 2011;16(11): $p=19820$. Available from: http://www.eurosurveillance.org/ViewArticle. aspx?Articleld $=19820$

17. Steens A, van der Hoek W, Dijkstra F, van der Sande M. Influenza vaccine effectiveness, 2010/11. Euro Surveill. 2011;16(15): $p=19843$. Available from: http://www. eurosurveillance.org/ViewArticle.aspx?Articleld=19843

18. Kissling E, Valenciano M, Cohen JM, Oroszi B, Barret AS, Rizzo C, et al. I-MOVE Multi-Centre Case Control Study 201011: Overall and Stratified Estimates of Influenza Vaccine Effectiveness in Europe. PLoS One. 2011;6(11):e27622.

19. Valenciano M, Kissling E, Ciancio BC, Moren A. Study designs for timely estimation of influenza vaccine effectiveness using European sentinel practitioner networks. Vaccine. 2010;28(46):7381-8.

20. Puig-Barbera J. 2010-2011 influenza seasonal vaccine, preliminary mid-season effectiveness estimates: reason for concern, confounding or are we following the right track? Euro Surveill. 2011;16(11): $p=19821$. Available from: http://www. eurosurveillance.org/ViewArticle.aspx?Articleld=19821

21. Kelly HA, Grant KA, Fielding JE, Carville KS, Looker CO, Tran T, et al. Pandemic influenza H1N1 2009 infection in Victoria, Australia: no evidence for harm or benefit following receipt of seasonal influenza vaccine in 2009 . Vaccine. 2011;29(37):6419-26.

22. Orenstein EW, De Serres G, Haber MJ, Shay DK, Bridges CB, Gargiullo $P$, et al. Methodologic issues regarding the use of three observational study designs to assess influenza vaccine effectiveness. Int J Epidemiol. 2007;36(3):623-31.

23. Lopez Chavarrias V, Broberg E, Nicoll A. Preliminary implications for Europe of the 2011 influenza season in five temperate southern hemisphere countries. Euro Surveill. 2011;16(50): $p=20044$. Available from: http://www. eurosurveillance.org/ViewArticle.aspx?Articleld=20044 